Editorial

\title{
Acknowledgment to Reviewers of Beverages in 2021
}

\section{Beverages Editorial Office}

MDPI AG, St. Alban-Anlage 66, 4052 Basel, Switzerland

Rigorous peer-reviews are the basis of high-quality academic publishing. Thanks to the great efforts of our reviewers, Beverages was able to maintain its standards for the high quality of its published papers. Thanks to the contribution of our reviewers, in 2021, the median time to first decision was 17 days and the median time to publication was 40 days. The editors would like to extend their gratitude and recognition to the following reviewers for their precious time and dedication, regardless of whether the papers they reviewed were finally published:

Agata Antoniewska

Agata Czyżowska

Aggelos Philippidis

Agnieszka Bem

Agustín V. Ruiz-Vega

Ainara López

Alan Gasiński

Alessandra Del Caro

Alessandro Bonadonna

Alexia N. Gloess

Amanda Dupas De Matos

Ana Cristina Correia

Ana Hranilovic

Ana Jeromel

Ana Jurinjak Tusek

Ana Kaic

Anamaria Calugar

Andreea Ratiu

Aneta Jastrzębska

Ángel Calín-Sánchez

Angel Carbonell-Barachina

Anita Oberholster

Anna Gramza-Michałowska

Anna Picinelli-Lobo

Anna Reale

Ante Galic

Antonietta Baiano

Antonio Domenico Marsico

Carlos Escott

Cecilia Roman

Charles Edwards

Chian Ju Jong

Chiara Nadai

Christian Philipp

Christopher Taylor
Clara Grosso

Corinna Hempel

Cristina Calvo Porral

Cristina Ubeda

Cynthia Whissell

Dawid Mikulski

Dirk Lachenmeier

Dirk W. Lachenmeier

Dolores González De Llano

Dominika Głąbska

Dominika Guzek

Dorota Derewiaka

Drew Budner

Durán-Guerrero Enrique

Elenilson De Godoy Alves Filho

Elvira Gaspar

Elwira Sieniawska

Emilia Janiszewska-Turak

Emilia Witkowska Nery

Fabio Chinnici

Félix Adjé

Fernanda Cosme

Fernanda Galgano

Fideline Laure Tchuenbou-Magaia

Francesco Barreca

Francesco Campa

Gary Pickering

Georgi Kostov

Gianfranco Pannella

Gianluca Paventi

Giovanni De Francesco

Glen Patrick Fox

Greta Adamczyk

Gustavo Gonzalez-Neves

Hanne Kristine Sivertsen 


\author{
Iain A. Brownlee \\ Ilda Caldeira \\ Isidora Radulov \\ Ivana Marová \\ Ivana Tomaz \\ Jana Okleštková \\ Jana Olsovska \\ Jawad Sarfraz \\ Jeanine Ammann \\ Jesús Ruiz-Nicolás \\ Joanna Chmielewska \\ Joanna Piepiórka-Stepuk \\ Jonathan Kershaw \\ José Sousa Câmara \\ Juan José Rodríguez-Bencomo \\ Julie Bower \\ Kadri Koppel \\ Kang-Mo Ku \\ Karoly Heberger \\ Katarzyna Świąder \\ Kathleen Kelley \\ Kavita Sharma \\ Kevin Kantono \\ Konstantinos Papadimitriou \\ Koushik Adhikari \\ Kristina Mastanjević \\ Krzysztof B. Beć \\ Lapo Pierguidi \\ Laura Grațiela Vicaș \\ Laura Stan \\ Leticia Martínez-Lapuente \\ Luca Altamore \\ Luigi Galletto \\ Luigino Barisan \\ Luisa Torri \\ Łukasz Wróblewski \\ Małgorzata Grembecka
}

Małgorzata Lasik-Kurdyś

Mani Naiker

Manohar Prasad Bhandari

Margarita A. Sazonova

Maria Carla Cravero

María Jesús Delgado Rodríguez

Maria João Barroca

Maria P. Serratosa

Marina V. Padkina

Mateusz Jackowski

Matteo Busconi

Matthew B. McSweeney

Ming-Kuei Shih

Mojtaba Kaviani

Monica Rosa Loizzo

Nicoleta Dospinescu

Olcay Kaplan Ince

Pasquale Russo

Patrizia Romano

Paweł Hanus

Paweł Kubica

Phil Bremer

Purificación Fernández-Zurbano

Rafael Apolinar-Valiente

Raul Ferrer-Gallego

Robert Brinson

Roberta Foligni

Romina Alina Marc

Rosa Perestrelo

Rosanna Tofalo

Rosario Sánchez-Gómez

Rositsa Stefanova Denkova-Kostova

Rui Manuel De Sousa Fragoso

Sabine Harrison

Sang-Han Lee

Stanislav Klazar

Wilfried Rozhon 